\title{
Local bifurcations of critical periods for quartic Liénard equations with quintic damping
}

\author{
Li Hongwei
}

Correspondence: If0539@126.com School of Science, Linyi University, Linyi 276005, Shandong, P.R. China

\begin{abstract}
In this article, we study the local bifurcation of critical periods near a nonde-generate center of the quartic Liénard equation with quintic damping and prove that at most two local critical periods can be produced from either a weak center of finite order or the isochronous center.

MSC: 34C05; 34C07.
\end{abstract}

Keywords: Liénard system, center, isochronous center, bifurcation of critical periods

\section{Introduction}

Liénard equation which contains planar Hamiltonian systems of Newton's type as a special case is one of the most important differential equations because it was widely used in physics and others. The theory of centers and isochronous centers of Liénard equation have been systematically investigated, but the theory of weak centers and local bifurcation of critical periods were developed slowly because computations are tedious and formidable.

In 1989, the theories of weak centers and local bifurcation of critical periods were investigated and applied to both quadratic Bautin's systems and planar Hamiltonian systems of Newton's type by Chicone and Jacobs [1]. Since then, great efforts have been made for systems of higher degree in the direction of quadratic Bautin's systems, see [2,3]. Meanwhile, great Efforts were also taken for some special systems, the reduced Kukles system was investigated by Rousseau and Toni [4] and reversible cubic perturbations of a quadratic isochronous center was studied by Zhang et al. [5]. On the other hand, many mathematicians have studied the weak centers and bifurcations of local critical period for Liénard equation $\ddot{x}+f(x) \dot{x}+g(x)=0$ in the direction of Chicone and Jacobs' study [1] on planar Hamiltonian systems, namely

$$
\begin{aligned}
& \frac{d x}{d t}=y, \\
& \frac{d y}{d t}=-g(x)-f(x) y .
\end{aligned}
$$

where $f, g$ are both polynomials. In this article, we assume that the equilibrium of interest is at the origin $O(0,0)$ which is nondegenerate. This requires $g(0)=0, f(0)=0$, $g^{\prime}(0)>0$. When $f, g$ are both quadratic polynomials, it has been studied carefully in [6]. Furthermore when $f, g$ are both cubic polynomials, they found that at most two local

(c) 2012 Hongwei; licensee Springer. This is an Open Access article distributed under the terms of the Creative Commons Attribution License (http://creativecommons.org/licenses/by/2.0), which permits unrestricted use, distribution, and reproduction in any medium, provided the original work is properly cited. 
critical periods can be produced from either a weak center of finite order or the linear isochronous center and that at most one local critical period can be produced from nonlinear isochronous centers in [7]. The quartic Liénard equation with quartic damping has been investigated by [8].

In this article, we assume

$$
f(x)=a_{1} x+a_{2} x^{2}+a_{3} x^{3}+a_{4} x^{4}+a_{5} x^{5}, g(x)=b_{1} x+b_{2} x^{2}+b_{3} x^{3}+b_{4} x^{4} .
$$

where the parameters $a_{1}, a_{2}, a_{3}, a_{4}, b_{1}, b_{2}, b_{3}, b_{4}, b_{5} \in R$ and $b_{1}>0$. This article will be organized as follows. In Section 2, we state some preliminary knowledge which is useful throughout the article. In Section 3, we first apply the results in $[9,10]$ on centers of polynomial Liénard equations to give a necessary and sufficient condition for a center at $O$ and find the set of coefficients in which the center is isochronous. Then in Section 4, we identify the weak centers of various possible order. This article is ended with Section 5 in which the local bifurcation of critical periods was discussed, the results that at most two local critical periods can be bifurcated from the linear or nonlinear isochronous center $O$ and for each $j \leq 2$ there is a perturbation with exactly $j$ local critical periods are proved.

\section{Preliminary knowledge}

In this section, we will recall the some related notions and results.

Let $P(r, \lambda)$ denotes the minimum period of the periodic orbit around the origin through a nonzero point $(r, 0)$. By the period coefficient lemma [1], $P(r, \lambda)$ is analytic locally and can be represented as its Taylor series $P(r, \lambda)=2 \pi+\sum_{k=2}^{\infty} p_{k}(\lambda) r^{k}$.

Definition 2.1. If there exists $\lambda^{*}=\left(a_{1}, a_{2}, a_{3}, a_{4}, a_{5}, b_{2}, b_{3}, b_{4}\right)$ such that $\tau_{2}\left(\lambda^{*}\right)=\cdots$ $=\tau_{2 k+1}\left(\lambda^{*}\right)=0$ and $\tau_{2 k+2}\left(\lambda^{*}\right) \neq 0$ for an integer $k$ then (3.1) has a weak center of order $k$ at $O$.

By the definition in [1], a local critical period is a period corresponding to a critical point of the period function $P(\cdot, \lambda)$ which arises from a bifurcation from a weak center. We say that $k$ local critical periods bifurcate from a weak center at $O$ corresponding to the parameter $\lambda^{*}$ if for every $\varepsilon>0$ and every neighborhood $W$ of $\lambda^{*}$ (in the region of parameters for which the system has a center at $O$ ) there is a point $\lambda^{1} \in W$ such that $P^{\prime}\left(r, \lambda^{1}\right)=0$ has $k$ solutions in $U=(0, \varepsilon)$. Moreover, we say that at most $k$ local critical periods bifurcate from a weak center at $O$ corresponding to the parameter $\lambda^{*}$ if for every $\varepsilon>0$ there is a neighborhood $W$ of $\lambda^{*}$ such that $P^{\prime}(r, \lambda)=0$ has $k$ solutions in $U$ $=(0, \varepsilon)$ for any $\lambda \in W$.

As defined in [11],

Definition 2.2. Real functions $g_{1}, \ldots, g_{l}$ on $R^{n}$ are said to be independent with respect to real function $g_{l+1}$ on $R^{n}$ at $\lambda^{* *} \in V\left(g_{1}, \ldots, g_{l}\right)$ if

(i) every open neighborhood of $\lambda^{*}$ contains a point $\lambda \in V\left(g_{1}, \ldots, g_{l-1}\right)$ such that $g_{l}(\lambda)$ $g_{l+1}(\lambda)<0$.

(ii) the varieties $V\left(g_{1}, \ldots, g_{j}\right), 2 \leq j \leq l-1$ are such that if $V\left(g_{1}, \ldots, g_{j}\right)$ and $g_{j+1}(\lambda) \neq$ 0 then every neighborhood $W$ of $\lambda$ contains a point $\sigma \in V\left(g_{1}, \ldots, g_{j-1}\right)$ such that $g_{j}(\lambda) g_{j}$ $+1(\lambda)<0$.

(iii) if $\lambda \in V\left(g_{1}\right)$ and $g_{2}(\lambda) \neq 0$, then every open neighborhood of $\lambda$ contains a point $\sigma$ such that $g_{1}(\sigma) g_{2}(\sigma)<0$. 
So it is easy to see that, if $g_{1}, \ldots, g_{l}$ are independent with respect to $g_{l+1}$ at $\lambda^{*} \in V$ $\left(g_{1}, \ldots, g_{l}\right)$ then, for each $k=2, \ldots, l, g_{1}, \ldots, g_{k-1}$ are independent with respect to $g_{k}$ at every $\lambda \in V\left(g_{1}, \ldots, g_{k-1}\right)$ such that $g_{k}(\lambda) \neq 0$.

Lemma 2.1. [9] System (1.1) with $f(x)=\sum_{i=1}^{m} a_{i} x^{i}, g(x)=\sum_{i=1}^{n} b_{i} x^{i}$, where $a_{i} b_{i} \in R$, $b_{1}=1$, has a center at $O$ if and only if

$$
\int_{0}^{x} f(\xi) d \xi=A(M(x)), \int_{0}^{x} g(\xi) d \xi=B(M(x)),
$$

for some polynomials $A, B$ and $M$ such that $M^{\prime}(0)=0, M^{\prime \prime}(0) \neq 0$

Lemma 2.2. [10]If $f(x)$ or $g(x)$ is odd, then $M=x^{2}$ and (1.1) has an isochronous center at the origin of (1.1) if and only if $f(x)$ is odd and

$$
g(x)=x+\frac{1}{x^{3}}\left(\int_{0}^{x} \xi f(\xi) d \xi\right)^{2}
$$

\section{Conditions for center and isochronous center}

We can always assume that $b_{1}=1$, unless we could make transformation $(x, t) \rightarrow$ $\left(x / \sqrt{b_{1}}, t / \sqrt{b_{1}}\right)$ to make $b_{1}=1$.

Applying Lemma 2.1 to the

$$
\begin{aligned}
& \frac{d x}{d t}=y, \\
& \frac{d y}{d t}=-x-b_{2} x^{2}-b_{3} x^{3}-b_{4} x^{4}-\left(a_{1} x+a_{2} x^{2}+a_{3} x^{3}+a_{4} x^{4}+a_{5} x^{5}\right) y .
\end{aligned}
$$

We give the following condition of center directly for coefficients.

Theorem 3.1. $O$ is a center of system (3.1) if and only if $\lambda \in S_{I} \cup S_{I I} \cup S_{I I}$, where

$$
\begin{gathered}
S_{I}=\left\{\lambda \in R^{8}: a_{2}=b_{2}=a_{4}=b_{4}=0\right\} ; \\
S_{I I}=\left\{\lambda \in R^{8}: a_{2}=a_{1} b_{2}, a_{4}=b_{4} a_{1}, a_{3}=a_{1} b_{3}, a_{5}=0\right\} ; \\
S_{I I I}=\left\{\lambda \in R^{8}: a_{2}=a_{1} b_{2}, a_{4}=\frac{5}{3} a_{3} b_{2}, a_{5}=\frac{2}{3} a_{3} b_{2}^{2}, b_{3}=b_{4}=0, b_{2} \neq 0\right\} .
\end{gathered}
$$

Proof. Our proof is based on the method developed by Cherkas [12]. In order to solve system (3.1) with $m=5, n=4$. we choose

$$
A(x)=\sum_{i=0}^{4} \alpha_{i} x_{i}, B(x)=\sum_{i=0}^{4} \beta_{i} x_{i}, M(x)=x^{2}+\sum_{i=3}^{6} m_{i} x_{i} .
$$

In fact,

$$
\int_{0}^{x} f(\xi) d \xi=\sum_{i=1}^{m} \frac{a_{i}}{i+1} x^{i+1}, \int_{0}^{x} g(\xi) d \xi=\sum_{i=1}^{n} \frac{b_{i}}{i+1} x^{i+1} .
$$

They are both polynomials of $M(x)$ if and only if they are both polynomials of polynomial $M(x) / m_{2}-m_{0} / m_{2}$, where $m_{0}, m_{1}$ are coefficients of the first two terms of polynomial $M(x)$. Without loss of generality, we can assume that $m_{0}=0, m_{2}=1$. 
Substituting those formal polynomials (3.2) in (2.1), and comparing coefficients with the help of mathematica, we obtain the conditions of theorem.

Theorem 3.2. $O$ is an isochronous center of system (3.1) if and only if $\lambda \in S_{I V}$, where

$$
S_{I V}=\left\{\lambda \in R^{8}: a_{2}=a_{3}=a_{4}=a_{5}=b_{2}=b_{4}=0, b_{3}=\frac{a_{1}^{2}}{9}\right\} .
$$

Proof. When $\lambda \in S_{I}$, Lemma 2.2 implies that system (3.1) has an isochronous center at $O$ if and only if

$$
x+b_{3} x^{3}=x+\frac{1}{x^{3}}\left(\frac{a_{1}}{3} x^{3}+\frac{a_{3}}{5} x^{5}+\frac{a_{5}}{7} x^{7}\right)^{2}
$$

Thus $b_{3}=\frac{a_{1}^{2}}{9}$ and $a_{3}=a_{5}=0$ implying that $\lambda L S_{I V}$

With the same method, we could get that $O$ is an isochronous center of system (3.1) when $\lambda \in S_{I}, S_{I I}, S_{I I I}$ if and only if $a_{1}=a_{2}=a_{3}=a_{4}=a_{5}=b_{1}=b_{2}=b_{3}=b_{4}=0$, namely $\dot{x}=-y, \dot{y}=x$. It imply that $\lambda \in S_{I V}$.

Obviously, $\lambda_{I} \cap \lambda_{I V}=\lambda_{I V}, \lambda_{I I} \cap \lambda_{I V}=(0,0,0,0,0,0,0,0), \lambda_{I I I} \cap \lambda_{I V}=(0,0,0,0,0,0,0$, $0)$, In the following section, we discuss the weak centers of finite order for $\left(\lambda_{I} \cup \lambda_{I I}\right.$ $\left.n \lambda_{I I I}\right)\left\langle\lambda_{I V}\right.$.

\section{Weak centers of finite order}

This section is devoted investigating how many local critical periods can be produced from a perturbed system of (3.1) near $O$. The independence condition should be used in the proof of bifurcation of critical periods. For more detail please see [11].

Theorem 4.1. $O$ is a weak center of order at most two of system (3.1) when $\lambda \in S_{I}$ $\mid S_{I V}$, and the center is of order $k(k=0,1,2,3)$ if and only if $\lambda \in \Lambda_{I}^{k}$, where

$$
\begin{gathered}
\Lambda_{I}^{0}=\left\{\lambda \in R^{8}: a_{2}=b_{2}=a_{4}=b_{4}=0, b_{3} \neq \frac{1}{9} a_{1}^{2}\right\} ; \\
\Lambda_{I}^{1}=\left\{\lambda \in R^{8}: a_{2}=b_{2}=a_{4}=b_{4}=0, b_{3}=\frac{1}{9} a_{1}^{2}, a_{1} a_{3} \neq 0\right\} ; \\
\Lambda_{I}^{2}=\left\{\lambda \in R^{8}: a_{1}=a_{2}=a_{4}=b_{2}=b_{3}=b_{4}=0, a_{3} \neq 0\right\} \cup \\
\left\{\lambda \in R^{8}: a_{3}=a_{2}=b_{2}=a_{4}=b_{4}=0, b_{3}=\frac{1}{9} a_{1}^{2}, a_{5} \neq 0\right\} .
\end{gathered}
$$

Proof. When $\lambda \in S_{I}$, with the computer algebra system Mathematic 8.0 we calculate

$$
\begin{aligned}
\tau_{1} & =\frac{1}{12}\left(9 b_{3}-a_{1}^{2}\right), \\
\tau_{2} & =-\frac{1}{12} a_{1} a_{3},
\end{aligned}
$$

If $a_{1}=0, a_{3} \neq 0$

$$
\tau_{3}=-\frac{7}{320} a_{3}^{2}
$$

If $a_{3}=0$,

$$
\tau_{3}=-\frac{5}{96} a_{5}^{2}
$$


So when $\tau_{1} \neq 0$, we have

$$
\Lambda_{I}^{0}=\left\{\lambda \in R^{8}: a_{2}=b_{2}=a_{4}=b_{4}=0, b_{3} \neq \frac{1}{9} a_{1}^{2}\right\} ;
$$

When $\tau_{1}=0, \tau_{2} \neq 0$, we have

$$
\Lambda_{I}^{1}=\left\{\lambda \in R^{8}: a_{2}=b_{2}=a_{4}=b_{4}=0, b_{3}=\frac{1}{9} a_{1}^{2}, a_{1} a_{3} \neq 0\right\}
$$

If $\tau_{1}=0, \tau_{2}=0, \tau_{3} \neq 0$, we have

$$
\begin{aligned}
\Lambda_{I}^{2}= & \left\{\lambda \in R^{8}: a_{1}=a_{2}=a_{4}=b_{2}=b_{3}=b_{4}=0, a_{3} \neq 0\right\} \cup \\
& \left\{\lambda \in R^{8}: a_{3}=a_{2}=b_{2}=a_{4}=b_{4}=0, b_{3}=\frac{1}{9} a_{1}^{2}, a_{5} \neq 0\right\} ;
\end{aligned}
$$

Theorem 4.2. $O$ is a weak center of order at most two of system (3.1) when $\lambda \in S_{I I}$ $\mid S_{I V}$, and the center is of order $k(k=0,1,2)$ if and only $\lambda \in \Lambda_{I I}^{k}$, where

$$
\begin{gathered}
\Lambda_{I I}^{0}=\left\{\lambda \in R^{8}: a_{2}=a_{1} b_{2}, a_{4}=b_{4} a_{1}, a_{3}=a_{1} b_{3}, a_{5}=0, b_{3} \neq \frac{1}{9}\left(a_{1}^{2}+10 b_{2}^{2}\right)\right\} ; \\
\Lambda_{I I}^{1}=\left\{\lambda \in R^{8}: a_{2}=a_{1} b_{2}, a_{4}=b_{4} a_{1}, a_{3}=a_{1} b_{3}, a_{5}=0,\right. \\
\left.b_{3}=\frac{1}{9}\left(a_{1}^{2}+10 b_{2}^{2}\right),-2 a_{1}^{4}+35 a_{1}^{2} b_{2}^{2}+280 b_{2}^{4}-378 b_{2} b_{4} \neq 0\right\} ; \\
\Lambda_{I I}^{2}=\left\{\lambda \in R^{8}: a_{2}=a_{1} b_{2}, a_{4}=b_{4} a_{1}, a_{3}=a_{1} b_{3}, a_{5}=0,\right. \\
\left.b_{3}=\frac{1}{9}\left(a_{1}^{2}+10 b_{2}^{2}\right), b_{4}=\frac{-2 a_{1}^{4}+35 a_{1}^{2} b_{2}^{2}+280 b_{2}^{4}}{378 b_{2}}, b_{2} \neq 0\right\} \cup \\
\left\{\lambda \in R^{8}: a_{1}=a_{2}=a_{3}=a_{4}=a_{5}=b_{2}=b_{3}=0, b_{4} \neq 0\right\}
\end{gathered}
$$

Proof. When $\lambda \in S_{I I}$, with the computer algebra system Mathematic 8.0 we calculate

$$
\begin{aligned}
& \tau_{1}=\frac{1}{12}\left(9 b_{3}-a_{1}^{2}-10 b_{2}^{2}\right) \\
& \tau_{2}=\frac{1}{216}\left(-2 a_{1}^{4}+35 a_{1}^{2} b_{2}^{2}+280 b_{2}^{4}-378 b_{2} b_{4}\right)
\end{aligned}
$$

If $b_{2}=0$,

$$
\tau_{2}=-\frac{a_{1}^{4}}{108}, \tau_{3}=-\frac{63}{80} b_{4}^{2}
$$

If $b_{2} \neq 0$,

$$
\tau_{3}=-\frac{12 a_{1}^{8}+987 a_{1}^{6} b_{2}^{2}+3395 a_{1}^{4} b_{2}^{4}+85750 a_{1}^{2} b_{2}^{6}+450800 b_{2}^{8}}{544320 b_{2}^{2}} .
$$

So when $\tau_{1} \neq 0$, we have

$$
\Lambda_{I I}^{0}=\left\{\lambda \in R^{8}: a_{2}=a_{1} b_{2}, a_{4}=b_{4} a_{1}, a_{3}=a_{1} b_{3}, a_{5}=0, b_{3} \neq \frac{1}{9}\left(a_{1}^{2}+10 b_{2}^{2}\right)\right\} ;
$$

When $\tau_{1}=0, \tau_{2} \neq 0$, we have

$$
\begin{aligned}
\Lambda_{I I}^{1}=\left\{\lambda \in R^{8}: a_{2}=a_{1} b_{2}, a_{4}=b_{4} a_{1}, a_{3}=a_{1} b_{3}, a_{5}=0,\right. \\
\left.\quad b_{3}=\frac{1}{9}\left(a_{1}^{2}+10 b_{2}^{2}\right),-2 a_{1}^{4}+35 a_{1}^{2} b_{2}^{2}+280 b_{2}^{4}-378 b_{2} b_{4} \neq 0\right\} ;
\end{aligned}
$$


If $\tau_{1}=0, \tau_{2}=0, \tau_{3} \neq 0$, we have

$$
\begin{aligned}
\Lambda_{I I}^{2}=\left\{\lambda \in R^{8}: a_{2}=a_{1} b_{2}, a_{4}=b_{4} a_{1}, a_{3}=a_{1} b_{3}, a_{5}=0,\right. \\
\\
\left.b_{3}=\frac{1}{9}\left(a_{1}^{2}+10 b_{2}^{2}\right), b_{4}=\frac{-2 a_{1}^{4}+35 a_{1}^{2} b_{2}^{2}+280 b_{2}^{4}}{378 b_{2}}, b_{2} \neq 0\right\} \cup \\
\left\{\lambda \in R^{8}: a_{1}=a_{2}=a_{3}=a_{4}=a_{5}=b_{2}=b_{3}=0, b_{4} \neq 0\right\}
\end{aligned}
$$

Theorem 4.3. $O$ is a weak center of order 0 of system (3.1) when $\lambda \in S_{I I I} \mid S_{I V}$.

Proof. When $\lambda \in S_{I I I}$, with the computer algebra system Mathematic 8.0 we calculate

$$
\tau_{1}=\frac{1}{12}\left(-a_{1}^{2}-10 b_{2}^{2}\right) \text {. }
$$

So $\tau_{1} \neq 0$, namely, the $O$ is a weak center of order 0 .

From Theorem 3.1 to 3.3 we conclude the following result.

Theorem 4.4. $O$ is a weak center of order at most 2 of system (3.1) when $\lambda \in\left(S_{I} \cup S_{I I}\right.$ $\left.\cup S_{I I I}\right) \backslash S_{I V}$, and the center is of order $k(k=1,2)$ if and only if $\lambda \in \Lambda_{I}^{k} \cup \Lambda_{I I}^{k}$, the center is of order 0 if and only if $\lambda \in \Lambda_{I}^{0} \cup \Lambda_{I I}^{0} \cup \Lambda_{I I I}^{0}$.

\section{Bifurcations of critical periods}

In this section, we investigate how many local critical periods can be produced from a perturbed system of (3.1) near $O$. The independence condition should be used in the proof of bifurcation of critical periods. For more detail please see [11].

Theorem 5.1. For each $k=1,2$ at most $k$ local critical periods occur in a perturbed system of (3.1) for $\lambda \in \Lambda_{I}^{k} \cup \Lambda_{I I}^{k}$. Moreover, there are perturbations of (3.1) where $\lambda \in \Lambda_{I}^{1} \cup \Lambda_{I I}^{1}$ with exactly one critical periods. There are perturbations of (3.1) where

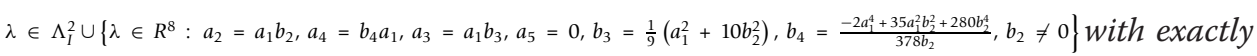
two critical periods.

Proof. The first assertion was directly proved by [1, Lemma 2.2], so we need only to check conditions for independence, in order to prove the second by [1, Theorem 2.1]. It is sufficient to discuss the case of $k=2$ and prove the independence of $\tau_{1}, \tau_{2}$ with respect to $\tau_{3}$ at each $\lambda \in \Lambda_{I}^{2} \cup \Lambda_{I I}^{2}$ by checking (i) and (iii) only.

$$
\text { Consider some } \quad \lambda^{*} \in \Lambda_{I}^{2} \cup \Lambda_{I I^{\prime}}^{2} \quad \text { when }
$$

$\lambda^{*} \in \Lambda_{I}^{2}$, if $\lambda^{*}=\left(0,0, a_{3}^{*}, 0, a_{5}^{*}, 0,0,0\right), a_{3}^{*} \neq 0$. It is obviously that every open neighborhood of $\lambda^{*}$ contains a point $\lambda_{1}=\left(-\operatorname{sgn}\left(a_{3}^{*}\right) \varepsilon, 0, a_{3}^{*}, 0, a_{5}^{*}, 0, \frac{\varepsilon^{2}}{9}, 0\right)$, where $\varepsilon$ $>0$ is sufficiently small. We can check that $\tau_{1}\left(\lambda_{1}\right)=0, \tau_{2}\left(\lambda_{1}\right) \tau_{3}\left(\lambda_{1}\right)<0$ for sufficiently small $\varepsilon$. If $\lambda^{*}=\left(a_{1}^{*}, 0,0,0, a_{5}^{*}, 0, \frac{\left(a_{1}^{*}\right)^{2}}{9}, 0\right), a_{5}^{*} \neq 0$. Obviously, every open neighborhood of $\lambda^{*}$ contains a point $\lambda_{1}=\left(a_{1}^{*}, 0,-\operatorname{sgn}\left(a_{1}^{*}\right) \varepsilon, 0, a_{5}^{*}, 0, \frac{\left(a_{1}^{*}\right)^{2}}{9}, 0\right)$, where $\varepsilon>0$ is sufficiently small. We can check that $\tau_{1}\left(\lambda_{1}\right)=0, \tau_{2}\left(\lambda_{1}\right) \tau_{3}\left(\lambda_{1}\right)<0$ for sufficiently small $\varepsilon$. Thus condition (i) of independence is checked for $\lambda^{*} \in \Lambda_{I}^{2}$.

When $\lambda^{*} \in \Lambda_{I I}^{2}$, If

$$
\lambda^{*}=\left(a_{1}^{*}, a_{1}^{*} b_{2}^{*}, a_{1}^{*} b_{3}^{*}, b_{4}^{*} a_{1}^{*}, 0, b_{2}^{*}, \frac{1}{9}\left(\left(a_{1}^{*}\right)^{2}+10\left(b_{2}^{*}\right)^{2}\right), \frac{-2\left(a_{1}^{*}\right)^{4}+35\left(a_{1}^{*}\right)^{2}\left(b_{2}^{*}\right)^{2}+280\left(b_{2}^{*}\right)^{4}}{378 b_{2}^{*}}\right), b_{2}^{*} \neq 0
$$


Obviously, every open neighborhood of $\lambda *$ contains a point

$$
\lambda_{1}=\left(a_{1}^{*}, a_{1}^{*} b_{2}^{*}, a_{1}^{*} b_{3}^{*}, b_{4}^{*} a_{1}^{*}, 0, b_{2}^{*}, \frac{1}{9}\left(\left(a_{1}^{*}\right)^{2}+10\left(b_{2}^{*}\right)^{2}\right), \frac{-2\left(a_{1}^{*}\right)^{4}+35\left(a_{1}^{*}\right)^{2}\left(b_{2}^{*}\right)^{2}+280\left(b_{2}^{*}\right)^{4}}{378 b_{2}^{*}}-\operatorname{sgn}\left(b_{2}^{*}\right) \varepsilon\right)
$$

where $\varepsilon>0$ is sufficiently small. After careful calculations, we can check that $\tau_{1}\left(\lambda_{1}\right)=$ $0, \tau_{2}\left(\lambda_{1}\right) \tau_{3}\left(\lambda_{1}\right)<0$ for sufficiently small $\varepsilon$. But $\lambda^{*}=\left(0,0,0,0,0,0,0, b_{4}^{*}\right), b_{4}^{*} \neq 0$. We could find that every open neighborhood of $\lambda^{*}$ do not contains a point $\lambda_{1}$ such that $\tau_{1}\left(\lambda_{1}\right)=0, \tau_{2}\left(\lambda_{1}\right) \tau_{3}\left(\lambda_{1}\right)<0$.

In order to check condition (iii), consider $\lambda \in V\left(\tau_{1}\right)$ and $\tau_{2}(\lambda) \neq 0$. By Theorem 1, it is equivalent to say $\lambda \in \Lambda_{I}^{1} \cup \Lambda_{I I}^{1} \quad$ Consider $\quad \lambda \in \Lambda_{I}^{1}$. If $\lambda^{*}=\left(a_{1}^{*}, 0, a_{3}^{*}, 0, a_{5}^{*}, 0, b_{3}=\frac{1}{9}\left(a_{1}^{*}\right)^{2}, 0\right), a_{1}^{*} a_{3}^{*} \neq 0$. Obviously, every open neighborhood of $\lambda^{*}$ contains a point $\lambda_{1}=\left(a_{1}^{*}-\operatorname{sgn}\left(a_{3}^{*}\right) \varepsilon, 0, a_{3}^{*}, 0, a_{5}^{*}, 0, \frac{\varepsilon^{2}}{9}, 0\right)$, where $\varepsilon>0$ is sufficiently small. We can check that $\tau_{1}\left(\lambda_{1}\right) \tau_{2}\left(\lambda_{1}\right)<0$ for sufficiently small $\varepsilon$. Thus condition (iii) of independence is checked for $\lambda^{*} \in \Lambda_{I}^{1}$.

Consider $\lambda \in \Lambda_{I I}^{1}$. If $\lambda^{*}=\left(a_{1}^{*}, a_{1}^{*} b_{2}^{*}, a_{1}^{*} b_{3}^{*}, b_{4}^{*} a_{1}^{*}, 0, b_{2}^{*}, \frac{1}{9}\left(\left(a_{1}^{*}\right)^{2}+10\left(b_{2}^{*}\right)^{2}\right), b_{4}^{*}\right)$, where $-2\left(a_{1}^{*}\right)^{4}+35\left(a_{1}^{*}\right)^{2}\left(b_{2}^{*}\right)^{2}+280\left(b_{2}^{*}\right)^{4}-378 b_{2}^{*} b_{4}^{*} \neq 0$. Obviously, every open neighborhood of $\lambda^{*}$ contains a point $\lambda_{1}=\left(\left(a_{1}^{*}, a_{1}^{*} b_{2}^{*}, a_{1}^{*} b_{3}^{*}, b_{4}^{*} a_{1}^{*}, 0, b_{2}^{*}, \frac{1}{9}\left(\left(a_{1}^{*}\right)^{2}+10\left(b_{2}^{*}\right)^{2}\right)+\operatorname{sgn}\left(-2\left(a_{1}^{*}\right)^{4}+35\left(a_{1}^{*}\right)^{2}\left(b_{2}^{*}\right)^{2}+280\left(b_{2}^{*}\right)^{4}-378 b_{2}^{*} b_{4}^{*}\right) \varepsilon, b_{4}^{*}\right)\right.$, where $\varepsilon>0$ is sufficiently small. We can check that $\tau_{1}\left(\lambda_{1}\right) \tau_{2}\left(\lambda_{1}\right)<0$ for sufficiently small $\varepsilon$. Thus condition (iii) of independence is checked for $\lambda^{*} \in \Lambda_{I I}^{1}$. Thus condition (iii) also holds.

As far as, we know that it is also interesting to investigate in local critical periods occurring from an isochronous center besides the bifurcations from weak centers of finite order. The study of critical period bifurcations from an isochronous center can be done only by investigating the algebraic structure of the ideal generated by all period coefficients, i.e., to find the basis of this ideal. As known in Theorem 2.2, $O$ is an isochronous center if and only if $\lambda \in S_{I V}$. In addition, $O$ is a linear isochronous center, when $a_{1}=0$ or nonlinear isochronous center when $a_{1} \neq 0$.

Theorem 5.2. For system (3.1), at most two local critical periods can be bifurcated from the linear isochronous center $O$ and for each $j \leq 2$ there is a perturbation with exactly j local critical periods; at most two local critical period can be bifurcated from the nonlinear isochronous center $O$ and there is a perturbation with each $j \leq 2$ local critical period.

Proof. Consider $O$ to be a linear isochronous center, namely $\lambda^{*}=(0,0,0,0,0,0,0$, $0)$, we first claim that at most two local critical periods bifurcate from $\lambda^{*}$. Assume that $k(k \geq 3)$ local critical periods bifurcate from $\lambda^{*}$ for every $\varepsilon>0$ and every neighborhood $W$ of $\lambda^{*}$ there is $\lambda_{1}$ such that equation $P^{\prime}\left(r, \lambda_{1}\right)=0$ has $k$ solutions in $(0, \varepsilon)$. Since $W \subset$ $S_{I} \cup S_{I I} \cup S_{I I I}$, by Theorem 1, for every $\lambda$ in $W$ the center is either of degree at most three or isochronous. It follows that the center corresponding to $\lambda_{1}$ cannot be isochronous but is of degree at most three, which implies that at most three local critical periods bifurcate, i.e., there is a neighborhood $B$ of $\lambda_{1}$ such that equation $P^{\prime}(r, \lambda)=0$ has at most three (other than $k$ ) solutions in $(0, \varepsilon)$ for any $\lambda \in B$, and we have proved that there is a perturbation with three local critical period. This contradiction proves our claim. 
Now, we prove that there is a perturbation of $\lambda *$ with $j \leq 2$ local critical periods. Obviously, every small neighborhood $W$ of $\lambda *$ contains a point of the form $(0,0, \delta, 0,0,0,0,0) \in \Lambda_{I}^{2}$, where $\delta>0$ is sufficiently small. By Theorem 1 , the system for $(0,0, \delta, 0,0,0,0,0)$ has a weak center of order two at $O$. Since, $W$ is also a neighborhood of $(0,0, \delta, 0,0,0,0,0)$, by Theorem 2 , for every $\varepsilon>0$ there is a point $\tilde{\lambda}$ in $W$ such that equation $P^{\prime}(r, \tilde{\lambda})=0$ has $j$ solutions in $(0, \varepsilon)$, implying that exact $j$ local critical periods bifurcate from $O$ corresponding to $\lambda *$. Thus, the first assertion is proved.

With the same method, consider $O$ to be a nonlinear isochronous center, namely $\lambda^{*}=\left(a_{1}, 0,0,0,0,0, \frac{a_{1}^{2}}{9}, 0\right)$. We find that in small neighborhoods of $\lambda *$ there are points in $\Lambda_{I}^{2}$ and in every neighborhoods of $\lambda^{*}$. There are points in $\Lambda_{I}^{1}$ in every neighborhoods of $\lambda *$, i.e., the origin becomes a weak center of order two. By Theorem 4.1, the second part of this theorem is proved.

\section{Remarks}

In this article, quartic Liénard equation with quintic damping are investigated. When $f$, $g$ are both quadratic polynomials, namely $a_{3}=a_{4}=a_{5}=b_{3}=b_{4}=0$, it has been studied carefully in [6]. Furthermore, when $a_{4}=a_{5}=b_{4}=0, f, g$ are both cubic polynomials, they found that at most two local critical periods can be produced from either a weak center of finite order or the linear isochronous center and that at most one local critical period can be produced from nonlinear isochronous centers in [7]. When $a_{5}=$ 0 , the quartic Liénard equation with quartic damping has been investigated by [8]. Our results cover results of above except when the origin is a isochronous center, we will investigate the algebraic structure of the ideal generated by all period coefficients in future.

\section{Acknowledgements}

This research was partially supported by the Nature Science Foundation of Shandong Province (ZR2010AL005).

\section{Competing interests}

The author declares that they have no competing interests.

Received: 4 November 2011 Accepted: 3 March 2012 Published: 3 March 2012

\section{References}

1. Chicone, C, Jacobs, M: Bifurcation of critical periods for plane vector fields. Trans Am Math Soc. 312, 433-486 (1989). doi:10.1090/S0002-9947-1989-0930075-2

2. Rousseau, C, Toni, B: Local bifurcation of critical periods in vector fields with homogenous nonlinearities of the third degree. Canad Math Bull. 36, 473-484 (1993). doi:10.4153/CMB-1993-063-7

3. Romanovski, VG, Han, M: Critical period bifurcations of a cubic system. J Phys A: Math Gen. 36, 5011-5022 (2003). doi:10.1088/0305-4470/36/18/306

4. Rousseau, C, Toni, B: Local bifurcation of critical periods in the reduced Kukles system. Canad Math Bull. 49, 338-358 (1997)

5. Zhang, W, Hou, X, Zeng, Z: Weak center and bifurcation of critical periods in reversible cubic systems. Comput Math Appl. 40, 771-782 (2000). doi:10.1016/50898-1221(00)00195-4

6. Dumortier, F, Li, C: Quadratic Lienard equations with quadratic damping. J Diff Equ. 139, $41-59$ (1997). doi:10.1006/ jeq.1997.3291

7. Zou, L, Chen, X, Zhang, W: Local bifurcations of critical periods for cubic Liénard equations with cubic damping. Comput Appl Math. 222, 404-410 (2008). doi:10.1016/j.cam.2007.11.005

8. $\mathrm{Xu}, \mathrm{Q}$, Huang, W: The center conditions and local bifurcation of critical periods for a Liénard system. Appl Math Comput. 217, 6637-6643 (2011). doi:10.1016/j.amc.2011.01.035

9. Christopher, C: An algebraic approach to the classification of centers in polynomial Liénard systems. J Math Anal Appl. 229, 319-329 (1999). doi:10.1006/jmaa.1998.6175 
10. Christopher, C, Devlin, J: On the classification of Liénard systems with amplitude-independent periods. J Diff Equ. 200, 1-17 (2004). doi:10.1016/j.jde.2004.01.008

11. Wang, Z, Chen, X, Zhang, W: Local bifurcations of critical periods in a generalized 2-D LV system. Appl Math Comput. 214(1):17-25 (2009). doi:10.1016/j.amc.2009.03.087

12. Cherkas, LA: Conditions for a center for the equation $P_{3}(x) y y^{\prime}=\sum_{i=0}^{2} P_{i}(x) y^{i}$. Diff Equ 10(2):367-368 (1974). (in Russian)

doi:10.1186/1687-1847-2012-24

Cite this article as: Hongwei: Local bifurcations of critical periods for quartic Liénard equations with quintic damping. Advances in Difference Equations 2012 2012:24

Submit your manuscript to a SpringerOpen ${ }^{\circ}$ journal and benefit from:

- Convenient online submission

- Rigorous peer review

- Immediate publication on acceptance

- Open access: articles freely available online

- High visibility within the field

- Retaining the copyright to your article

Submit your next manuscript at $\boldsymbol{s p r i n g e r o p e n . c o m ~}$ 